PSFC/JA-06-9

\title{
Proton core imaging of the nuclear burn in inertial confinement fusion implosions
}

\author{
J. L. DeCiantis, ${ }^{1}$ F. H. Séguin, ${ }^{1}$ J. A. Frenje, ${ }^{1}$ V. Berube, ${ }^{1}$ M. J. \\ Canavan, ${ }^{1}$ C. D. Chen, ${ }^{1}$ S. Kurebayashi, ${ }^{1}$ C. K. Li, ${ }^{1}$ J. R. \\ Rygg, ${ }^{1}$ B. E. Schwartz, ${ }^{1}$ R. D. Petrasso ${ }^{1}$, J. A. Delettrez, ${ }^{2}$ S. P. \\ Regan, ${ }^{2}$ V. A. Smalyuk, ${ }^{2}$ J. P. Knauer, ${ }^{2}$ F. J. Marshall, ${ }^{2}$ D. D. \\ Meyerhofer ${ }^{2}$, S. Roberts, ${ }^{2}$ T. C. Sangster, ${ }^{2}$ C. Stoeckl ${ }^{2}$, K. \\ Mikaelian, ${ }^{3}$ H. S. Park, ${ }^{3}$ H. F. Robey ${ }^{3}$
}

\footnotetext{
${ }^{1}$ Plasma Science and Fusion Center, Massachusetts Institute of Technology, Cambridge, Massachusetts, 02139

${ }^{2}$ Laboratory for Laser Energetics, University of Rochester, Rochester, New York, 14623

${ }^{3}$ Lawrence Livermore National Laboratory, Livermore, California 94550
}

1 April 2006

This work was supported in part by U.S. Department of Energy Contract \#DEFG03-99SF21782, LLE subcontract \#PO410025G, and LLNL subcontract \#B313975. 


\title{
Proton core imaging of the nuclear burn in inertial confinement fusion implosions
}

\author{
J. L. DeCiantis, F. H. Séguin, ${ }^{a)}$ J. A. Frenje, V. Berube, M. J. Canavan, C. D. Chen, \\ S. Kurebayashi, C. K. Li, J. R. Rygg, B. E. Schwartz, R. D. Petrasso ${ }^{\text {b) }}$ \\ Plasma Science and Fusion Center, Massachusetts Institute of Technology, \\ Cambridge, Massachusetts, 02139
}

J. A. Delettrez, S. P. Regan, V. A. Smalyuk, J. P. Knauer, F. J. Marshall, D. D. Meyerhofer ${ }^{\text {c) }}$, S. Roberts, T. C. Sangster, C. Stoeckl

Laboratory for Laser Energetics, University of Rochester, Rochester, New York, 14623

K. Mikaelian, H. S. Park, H. F. Robey

Lawrence Livermore National Laboratory, Livermore, California 94550

\begin{abstract}
A proton emission imaging system has been developed and used extensively to measure the nuclear burn regions in the cores of inertial confinement fusion implosions. Three imaging cameras, mounted to the 60-beam OMEGA laser facility [T. R. Boehly et al., Opt. Commun. 133, 495 (1997)], use the penetrating $14.7-\mathrm{MeV}$ protons produced from $\mathrm{D}^{3} \mathrm{He}$ fusion reactions to produce emission images of the nuclear burn spatial distribution. The imaging technique relies on penumbral imaging, with different reconstruction algorithms for extracting the burn distributions of symmetric and asymmetric implosions. The hardware and design considerations required for the imaging cameras are described. Experimental data, analysis, and error analysis are presented for a representative symmetric implosion of a fuel capsule with a $17-\mu \mathrm{m}$-thick plastic shell and 18 -atm $\mathrm{D}^{3} \mathrm{He}$ gas fill. The radial burn profile was found to have characteristic radius $R_{\text {burn, }}$, which we define as the radius containing half the $\mathrm{D}^{3} \mathrm{He}$ reactions, of $32 \pm 1.6 \mu \mathrm{m}$ (burn radii measured for other capsule types range from 20 to $80 \mu \mathrm{m}$ ). Potential sources of error due to proton trajectory changes from interactions with electric fields and scattering in capsule and camera hardware are estimated with simple analytic and Monte Carlo calculations; they are predicted to be small compared with statistical errors. Experimental tests were performed to look for any inconsistencies between results from different cameras and different imaging geometries, or evidence of error due to ambient electric or magnetic fields, and none were found.
\end{abstract}

a)seguin@mit.edu

b) Also Visiting Senior Scientist, Laboratory for Laser Energetics, University of Rochester.

c) Also Departments of Mechanical Engineering, Physics and Astronomy, University of Rochester.

Byline: Proton core imaging for ICF 


\section{INTRODUCTION}

Images of the nuclear burn region in inertial confinement fusion (ICF) experiments ${ }^{1,2}$ can provide critical information about the complex dynamics of fuel capsule implosions, showing the spatial distributions of nuclear reactions that result from the cumulative effects of drive and compression (including fuel-shell mix, implosion asymmetries, and radiation and heat transport). Such image data provide exacting tests for simulations. Burn images of deuterium-tritium-filled capsules have previously been envisioned ${ }^{3}$ and made using $14.1-\mathrm{MeV}$ neutrons, ${ }^{4-7} 3-\mathrm{MeV}$ protons $^{8-10}$ or $3.5-\mathrm{MeV}$ alpha particles ${ }^{10}$ (see also other papers cited in Ref. 11).

This article is the second in a series that discusses another burn imaging method based on the energetic $14.7-\mathrm{MeV}$ protons from reactions in fuel with deuterium (D) and 3-helium $\left({ }^{3} \mathrm{He}\right)$ fuel ions. This method, utilizing multiple proton emission cameras, allows us to study the sizes and asymmetries of burn regions in a wide range of imploded thick-plastic-shell capsules that are not amenable to imaging with lower-energy charged particles (which cannot penetrate the shell) and with more angular views than have been obtained so far with neutron imaging. The first article $^{11}$ described the general method, presented the mathematical algorithms used, and evaluated the spatial resolution and signal-to-noise issues. Here we describe details of the imaging hardware, a sample analysis of a radial burn profile measurement, and experiments and calculations that have been performed to test the accuracy of the measurements. In other articles we show systematic studies of the effects of capsule and drive conditions on burn region size for a large number of nominally-symmetric implosions ${ }^{12,13}$ and the effects of drive asymmetries and capsule shell asymmetries on implosion asymmetry. ${ }^{14}$

The proton-emission imaging cameras generate penumbral images of the burn region that are deconvolved using the methods described in refs. 11 and 12. Two separate approaches are utilized; one generates a two dimensional (2-D) surface-brightness image of the burn, while the other generates a radial burn profile (reactions per unit volume) for nominally symmetric implosions. The work described here uses only the second approach.

The structure of this paper is as follows. Section II describes the camera hardware, which consists primarily of an imaging aperture and a detector pack utilizing CR-39 solid-state nuclear track detectors. Section III shows sample raw experimental data obtained from an implosion, along with the corresponding deconvolved radial profile of emissivity in the imploded capsule. Section IV investigates four possible sources of image degradation, all of which are shown to be unimportant. Section V discusses several experimental demonstrations of the fidelity of the reconstructed images, and Sec. VI summarizes the main results.

\section{HARDWARE AND DESIGN FEATURES}

Three identical Proton Core Imaging System (PCIS) cameras have been fabricated and used simultaneously for imaging $\mathrm{D}^{3} \mathrm{He}$ burn from three (nearly) orthogonal directions at the OMEGA laser facility. ${ }^{15}$ Design criteria for individual cameras included flexibility in imaging geometry and in detector filter assignments for imaging of various implosion types; modularized design for simplified installation and minimization of turn-around time between successive experiments; and a redundant "dual detection" scheme for verifying results and for backup in case of defective detectors.

The integrated system has proven very practical and effective for obtaining burn images at OMEGA. Figure 1 shows a schematic of three proton-emission imaging cameras mounted on 
the OMEGA target chamber, where each camera is positioned by a special diagnostic shuttle system. The simultaneous use of three cameras makes possible studies of burn symmetry ${ }^{11,14}$ and of camera consistency (see Sec. V). The structure of an individual camera is shown in Fig. 2.

After leaving an imploded capsule, protons pass through a round aperture before striking a detector pack. The aperture is typically a $2000-\mu \mathrm{m}$-diameter hole machined into a $500-\mu \mathrm{m}-$ thick tantalum (Ta) substrate, although diameters as small as $500 \mu \mathrm{m}$ have occasionally been used. An accurate, clean, and burr-free edge for the aperture wall is obtained using electrodischarge machining. Each aperture is inspected and quantitatively characterized on both sides using a microscope with a calibrated $\mathrm{X}-\mathrm{Y}$ stage and a high-resolution camera in order to determine that deviations from roundness are small; going around the circumference, the rms deviation from constant radius is not allowed to exceed $3 \mu \mathrm{m}$ and is usually considerably smaller.

The distance of the aperture from the imploded capsule, which is located at target chamber center (TCC), is usually as small as possible $(\sim 3 \mathrm{~cm})$ to maximize counting statistics; ${ }^{11}$ closer placement is not possible without interfering with laser beams. The region between the aperture and the detector is enclosed within a conductive housing (Fig. 2a). This enclosure prevents stray charged particles from reaching the detectors and shields signal protons from possible electric and magnetic fields.

The detector pack consists of a layered assembly of metallic foils and CR-39 detectors ${ }^{16}$ held in an aluminum (Al) support. The metallic foils act as ranging filters for reducing the energy of incoming protons to the energy range 0.5 to $8 \mathrm{MeV}$, where CR-39 proton detection efficiency is essentially $100 \%$. After exposure, each detector is etched in $\mathrm{NaOH}$ and scanned on an automated microscope system that locates the track of each incident proton to submicron accuracy. The distribution of proton track density on the detector forms the penumbral image referred to above.

The choice of filters depends on the expected proton spectrum, which can be different for different types of capsule implosions. Figure 3 shows spectra for a capsule with a 20 - $\mu$ m-thick plastic shell and a capsule with a 2 - $\mu \mathrm{m}$-thick glass-shell capsule; the mean proton energies were $13 \mathrm{MeV}$ and $14.5 \mathrm{MeV}$, respectively. The plastic-shell implosion might be imaged using a single $800-\mu \mathrm{m}$ thick Al filter, while the glass-shell implosion might require a $1000-\mu \mathrm{m}$ filter. Rather than a single filter, a series of filters and sheets of CR-39 are usually stacked to provide two redundant images from a single camera. As illustrated in Fig. 4a, two images are obtained when a thick filter is placed in front of two back-to-back sheets of CR-39. The filter and first sheet of CR-39 range down the energetic $\mathrm{D}^{3} \mathrm{He}$ protons for optimal detection on the back of the first sheet and again on the front of the second sheet. The redundant data are occasionally useful if one CR-39 detector has any defects (such as anomalous intrinsic noise ${ }^{16}$ ). Figure $4 \mathrm{~b}$ illustrates another filter configuration that can be used for redundant data. This one places a thin filter between the two pieces of CR-39 so that the proton energy is slightly different at the two detector surfaces. This can be useful if there is a significant uncertainty in what the proton energy will be or if the proton spectrum is particularly wide; if the spectrum at the first sheet is not optimum for detection, the spectrum at the other sheet may be better.

The distance from the aperture to the detector pack can be varied by positioning the detector in any of three designated slots (shown in Fig. 2a). The first slot provides a nominal 12.5x geometric magnification $M$ (the ratio $L_{2} / L_{1}$ ), the second provides $16 \mathrm{x}$, and the third provides $20 x$. The position is usually chosen to optimize the density of proton tracks on the 
detectors, which begin to saturate if the incident proton density exceeds $\sim 10^{6}$ protons $/ \mathrm{cm}^{2}$. The third slot makes possible the formation of images for $\mathrm{D}^{3} \mathrm{He}$ yields up to $\sim 5 \times 10^{10}$.

Each camera is secured to a ten-inch manipulator (TIM) on the OMEGA target chamber by way of the large, flat structure at the back end of the system (Fig. 2). The TIMs are diagnostic shuttle systems used to position diagnostics near the implosion. There are six TIMs on the OMEGA target chamber, allowing for the use of multiple PCIS cameras. The TIMs facilitate the installation, positioning, and removal of the cameras. Ease of access is essential since the aperture and detector pack of each camera must be replaced between every laser shot. Modularized aperture and detector packs have been implemented to ensure that the replacement time does not exceed the typical shot-to-shot time interval of the OMEGA laser.

\section{SAMPLE DATA FROM A CAPSULE WITH A PLASTIC SHELL}

The proton-emission imaging cameras have been used to examine many implosions at the OMEGA laser system; the majority of these involved direct illumination of warm plastic-shell capsules filled with a $\mathrm{D}^{3} \mathrm{He}$ gas mixture. These capsules were usually driven by 60 beams of frequency-tripled $(0.35-\mu \mathrm{m}) \mathrm{UV}$ light, with total laser energy of $23 \mathrm{~kJ}$ delivered in a 1-ns square pulse. $^{17}$ Individual beams underwent $1.0-\mathrm{THz} 2-\mathrm{D}$ smoothing by spectral dispersion and polarization smoothing applied through the use of birefringent wedges. ${ }^{18}$

Structural information about the source of protons in an experiment is obtained by deconvolving a penumbral camera image using the method described in ref. 11 and expanded in Ref. 12, which gives the time-integrated radial burn profile (reactions per $\mu \mathrm{m}^{3}$ ) in the implosion. First the azimuthally averaged proton density $N(R)$ in the penumbral image is used to calculate the radial derivative $\mathrm{d} N / \mathrm{d} R$, as illustrated in Fig. 5. An example using real data is shown in Fig. 6a (from OMEGA implosion 36730, with 18 -atm of $\mathrm{D}^{3} \mathrm{He}$ in a $17-\mu \mathrm{m}$-thick plastic shell). This derivative is then fit with a family of functions of varying shapes that are analytically or numerically related to functions describing the radial profile $S_{b u r n}(r)$ of proton emissivity per unit volume. The result is a determination of the shape and the characteristic size of the burn region itself. The size can be quantified in different ways, but the radius $R_{\text {burn }}$ containing $1 / 2$ of the total local emission seems quite independent of the emission profile shape (as discussed in ref. 12) and will be used here. For local emission profile shapes we can use the family of superGaussians

$$
S_{\text {burn }}(r)=S_{0} \exp \left[-\left(r / r_{0}\right)^{2 / p}\right]
$$

where $p$ is a "peakedness" shape parameter and $r_{0}=r_{0}\left(R_{b u r n}, p\right)$ is a radius. Numerically converting $S_{\text {burn }}(r)$ to functions for comparison with $\mathrm{d} N / \mathrm{d} R$, we find the best fit illustrated in Fig. $6 \mathrm{a}$; the corresponding radial profile of emissivity in the capsule is shown in Fig. $6 \mathrm{~b}$, and has $R_{\text {burn }}$ $=32 \mu \mathrm{m}$. To give an idea of statistical errors, Fig. $6 \mathrm{~b}$ shows emission profiles corresponding to

alternative fits to the raw data with total $\chi^{2}$ values higher than the minimum by one. The uncertainty in the shape is significant, but the uncertainty in $R_{b u r n}$ is only about $1.6 \mu \mathrm{m}$. Note that the analysis produces absolutely normalized emissivity values.

The $\mathrm{D}^{3} \mathrm{He}$ burn data from many nominally symmetric implosions have been characterized in this way. A range of $R_{\text {burn }}$ values from 20 to $80 \mu \mathrm{m}$ has been measured in other experiments, demonstrating the applicability of this imaging technique to widely different implosion types. The dependence of $R_{\text {burn }}$ on implosion type is discussed in Ref. 12. 


\section{DEMONSTRATION THAT POSSIBLE SOURCES OF IMAGE BROADENING ARE UNIMPORTANT}

As illustrated in Sec. III, statistical errors in the measurement of burn region size are typically a few $\mu \mathrm{m}$. In this section we investigate other possible sources of error. The protons that generate a penumbral image may experience trajectory shifts while traversing electric and magnetic fields and while interacting with different materials such as the capsule shell, the aperture substrate, and the ranging filters. If these trajectory shifts are severe enough, the spatial structure in the penumbral image may be artificially broadened; the structure in deconvolved burn images or radial burn profiles will therefore also be broadened. The azimuthal average of the penumbra can also be broadened if the aperture is not round, and errors in analysis can occur from use of incorrect geometric parameters. If broadening with a characteristic length $l$ occurs in the penumbral image, the deconvolved image of a point source (the point response function $\mathrm{prf}$ ) will have a characteristic size $l / M$, where $M$ is the geometric magnification of the camera (the value 12.5 is used in the following calculations). The effects of trajectory shifts are discussed below in order of occurrence, beginning at the location of $\mathrm{D}^{3} \mathrm{He}$ proton production and ending at the detector, and are followed by a consideration of errors in system geometry.

\section{A. Scattering in the compressed capsule}

The first significant materials encountered by the protons are the remnants of the shell that surround and compress the heated fuel region (see Fig. 7). Since the majority of implosions imaged with PCIS involved 20- $\mu$ m-thick CH-plastic shells, this type of capsule will be examined. At the time of peak proton production, the shell material remaining after the ablation phase consists of a highly dense $(\sim 20 \mathrm{~g} / \mathrm{cc})$, low-temperature $(\sim 500 \mathrm{eV})$ plasma. ${ }^{19,20}$ Spectral analysis of $\mathrm{D}^{3} \mathrm{He}$ fusion protons has shown that the shell material, with a corresponding $\rho \mathrm{R}$ of $\sim$ $60 \mathrm{mg} / \mathrm{cm}^{2}$, is responsible for a downshift of a couple $\mathrm{MeV}$ from the 14.7-MeV birth energy (Fig. 3a). ${ }^{21,22}$ Although proton slowing down is associated with drag of the background electrons, a small amount of scattering will occur off background ions. To estimate the amount of this scattering and its effect on the broadening of the prf of the imaging system, two separate calculations were performed.

In the first calculation, the shell material was treated as a cold, dense solid. The Monte Carlo code TRIM ${ }^{23}$ was used to simulate the slowing down and scatter of $14.7-\mathrm{MeV}$ protons in the cold shell material. The shell was treated as a 50/50 atomic mixture of carbon and hydrogen with a density of $20 \mathrm{~g} / \mathrm{cc}$ and a thickness of $35 \mu \mathrm{m}\left(\mathrm{a} \rho \mathrm{R}\right.$ of $70 \mathrm{mg} / \mathrm{cm}^{2}$ ). The calculated average transmitted energy was $12.2 \mathrm{MeV}$, in reasonable agreement with experimental observations (Fig. $3 a)$. The average scattering angle was found to be $0.9^{\circ}$. Assuming the fuel-shell interface is at a radius of $45 \mu \mathrm{m}$ and all scattering occurs on the outside edge of the shell, a worst-case amount of apparent source broadening was estimated to be $\sim 1 \mu \mathrm{m}$ (see Fig. 7).

In the second, more realistic calculation, the compressed shell material was treated as hot plasma at a temperature of $500 \mathrm{eV} \cdot{ }^{19,20}$ At this temperature and density, the shell atoms are fully ionized. $^{24}$ With minor modifications to Rutherford's scattering formula, ${ }^{25}$ the mean squared angle of the scattered particles is given by

$$
\left\langle\theta^{2}\right\rangle \cong 2 \pi N_{i}(2 z e Z e / p v)^{2}(\ln \Lambda) t
$$


where $N_{i}$ is the ion species density in the background plasma, $t$ is the shell thickness, and $Z e$ is the charge of the scattering material; $z e$ is the charge of the incident particle, with momentum $p$, and speed $v$; and $\ln \Lambda$ is the Coulomb logarithm. The mean scattering angle was calculated for $12-\mathrm{MeV}$ protons that have passed through $35 \mu \mathrm{m}$ of a $500 \mathrm{eV}, 20 \mathrm{~g} / \mathrm{cc} \mathrm{CH}$ plasma with a Coulomb logarithm of approximately $13 .{ }^{19,20}$ (An initial energy of $12 \mathrm{MeV}$ and a $\rho \mathrm{R}$ of 70 $\mathrm{mg} / \mathrm{cm}^{2}$ were selected to overestimate the effect.) The calculations gave an average scatter angle of $\sim 2$ degrees, corresponding to a broadening of the $\operatorname{prf}$ by $\sim 3 \mu \mathrm{m}$. (A similar calculation was performed for scattering in the fuel region, but the effect was much smaller.)

\section{B. Trajectory shifts due to electric fields}

During the laser pulse of a direct-drive implosion, hot electrons are blown off the capsule and may redeposit themselves on surrounding structures, temporarily establishing electric fields. The purpose of this section is to examine the effect a potential difference between the capsule and the aperture could have on proton trajectories and ultimately on the prf of the instrument. Although the effect is complicated by the structure of the OMEGA target chamber and the dynamics of the implosion, these calculations look at simplified geometries to produce an order of magnitude estimate of the effect.

A program was developed for tracking the trajectories of $14.7-\mathrm{MeV}$ protons emitted from a point source as they pass through a negatively charged aperture substrate and on to the CR-39 detector plane. A diagram of the geometry is shown in Fig. 8a. The aperture was positioned 3.3 $\mathrm{cm}$ from the source, and the detector plane was positioned an additional $33 \mathrm{~cm}$ past the aperture. The figure illustrates one possible effect of a charged aperture, where a proton path is indicated for two scenarios: for the case of a charged aperture (dashed line), and the case of an uncharged aperture (solid line).

The aperture substrate was modeled by a 1-cm-radius disk carrying a uniform negative charge corresponding to a potential between the source and this disk of $1 \mathrm{MV}$. The magnitude of the potential was chosen to overestimate experimental values expected. Experiments indicate that the capsule itself may carry a charge of approximately $1 \mathrm{MV}$ during the laser pulse, but that

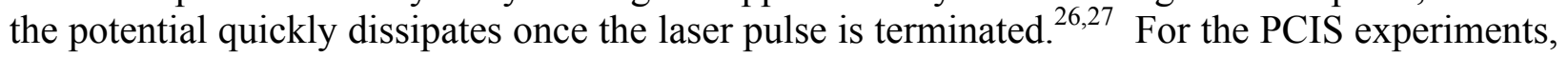
the proton production occurs several hundred picoseconds after laser pulse is terminated, providing a significant period of time in which the potential is known to have substantially decayed.

Calculations were performed for a 400- $\mu$ m-diameter aperture located in the center of the $1-\mathrm{cm}$ radius disk. Protons were launched through the aperture in all allowable directions. The code calculated particle forces and trajectories on a time scale corresponding to a $5-\mu \mathrm{m}$ step in the linear dimension using a standard integration for the velocity and a second-order RungeKutta integration for the radial position. Simulations were performed with the electric field symmetric on both sides of the aperture as well as with the field turned off between the aperture and the detector, modeling the limit of a perfectly conductive cavity enclosing the instrument. Particle trajectories were individually tracked and particle-particle interactions were not included, as the particle density in the experiments is small. The simulated proton density striking the detector is shown in Fig. $8 \mathrm{~b}$ for the case of symmetric fields. The solid line represents the proton density when no net potential exists between the source and the aperture, and the dashed line represents the proton density for a 1-MV potential. 
The purpose of this study was to estimate the effect of a potential difference between the aperture and the capsule on the prf of the camera. For both symmetric and asymmetric fields, the prf was found to be broadened by only $\sim 1 \mu \mathrm{m}$. (Additional simulations were also performed for a $1000-\mu \mathrm{m}$-diameter aperture and for a $0.1-\mathrm{MV}$ potential. None of the studies produced a significant broadening of the image, and the amount of broadening was independent of aperture size.)

\section{Scattering in aperture walls}

One advantage of proton-emission imaging is that a thin aperture substrate is sufficient to stop $\mathrm{D}^{3} \mathrm{He}$ protons, providing a well-defined aperture edge. The apertures commonly used consist of a $2000-\mu \mathrm{m}$-diameter cylindrical hole in a 500- $\mu$ m-thick Ta substrate. The $14.7-\mathrm{MeV}$ protons would be completely stopped by $\sim 400 \mu \mathrm{m}$ of Ta. Protons that travel through less Ta may be stopped in the ranging filters located in the detector pack. Ideally, no detected protons would have scattered in the Ta. Since no aperture is perfectly tapered to match the trajectories of incoming protons, however, some protons will strike the inner walls of the aperture and scatter through a thinner section of Ta, as illustrated in Fig. 9a. If the section is thin enough, protons may exit the opposite side, travel to the detector pack, pass through the ranging filters, and be detected on the CR-39. The result would be a slight broadening of the penumbra.

Simulations were performed to estimate the degradation of the camera prf by proton scattering in the Ta substrate utilizing the Monte Carlo program TRIM $^{23}$ to evaluate the slowing down and scattering of charged particles in matter. The source was treated as a $12-\mathrm{MeV}$ monoenergetic point source of protons, chosen to approximate the observed mean energy of $\mathrm{D}^{3} \mathrm{He}$ protons after escaping an imploded $20-\mu \mathrm{m}-\mathrm{CH}$-shell capsule. TRIM was used to determine the amount of scatter produced by discrete thicknesses of Ta; since the aperture wall presents a continuous range of thicknesses to protons, some assumptions were made to transform the 1-D TRIM output into the 2-D geometry of the aperture. Instead of modeling the continuous range of thicknesses, a series of seven discrete thickness steps was used. Depending on where the proton entered the aperture wall, the encountered material was approximated by one of seven Ta sheets, ranging in thickness from $10 \mu \mathrm{m}$ to $150 \mu \mathrm{m}$. (Any proton passing through more than $150 \mu \mathrm{m}$ of Ta would be stopped by the ranging filters.) Several million protons were tracked through each discrete thickness. Upon exiting the Ta material, the energy and trajectory of each proton was recorded.

These trajectories, along with the system geometry, were used to predict whether scattered protons would strike the detector plane. The system geometry mimicked the actual experimental hardware; modeling of the three main components, the source, the aperture, and the detector was included. The geometry was similar to that seen in Fig. 2b, where the distance from TCC to the aperture was taken to be $3 \mathrm{~cm}$ and the distance from the aperture to the detector was $33 \mathrm{~cm}$. The aperture was modeled by a $2000-\mu \mathrm{m}$ diameter hole centered in a $500-\mu \mathrm{m}$ thick Ta disk. Based on this geometry and the recorded trajectories from TRIM, the scattered protons that reached the detector pack were isolated from those that did not.

Each proton deflected toward the detector pack then encountered additional filtering before reaching the detector. The filtering, placed directly in front of the detector, was assumed to be $20 \mu \mathrm{m}$ of $\mathrm{Al}$ followed by $1000 \mu \mathrm{m}$ of Mylar and an additional $100 \mu \mathrm{m}$ of Al (Mylar has chemical composition and stopping properties similar to those of CR-39). A proton was assumed detected if its energy was great enough to allow passage through these additional filters. 
Thus, a record of the detection locations of all the scattered protons with an appropriate trajectory and energy was created. The ratio of the scattered proton density to unscattered signal proton density as a function of the detector radius is shown in Fig. 9b; the dotted lines indicate the approximate location of the image penumbra from a $30-\mu \mathrm{m}$ source. The simulations showed that the scattered protons represent less than $0.3 \%$ of the detected protons in the penumbral region and that the radial profile of scattered protons is fairly flat. Since the radial derivative of the proton density ultimately determines the size and shape of the source, ${ }^{11}$ the small-amplitude, slowly varying background would have little effect. Broadening of the prf was found to be $<<1$ $\mu \mathrm{m}$.

\section{Scattering in filters}

Upon reaching the detector pack, protons pass through several ranging filters that are designed to reduce the incident proton energy for optimal detection efficiency. Along with the desired loss of energy, the protons will experience some lateral straggling, another possible source of spatial broadening. To estimate the magnitude of this effect, the Monte Carlo code TRIM was used to track 12-MeV protons from a point source through $20 \mu \mathrm{m}$ of Al, $1000 \mu \mathrm{m}$ of Mylar, and a final $100 \mu \mathrm{m}$ of Al. The lateral positions for protons exiting the filters were recorded and fit by a Gaussian distribution with an e-folding radius at the detector of $\sim 30 \mu \mathrm{m}$. This translates to a broadening in the camera $\operatorname{prf}$ of $\sim 30 \mu \mathrm{m} / M \sim 3 \mu \mathrm{m}$.

\section{E. Geometric error}

Accuracy in the inference of burn profiles from penumbral images requires accurate knowledge of imaging system dimensions. Of primary importance is the radius of the aperture projection on the detector, which may not be exactly as expected if either the capsule-aperture distance or the aperture-detector distance has any uncertainty. But the radius is actually determined from the data as an integral part of the analysis (see Refs. 11 and 12 and Fig. 6a), so this is not a source of error beyond the statistical fit error. If the aperture itself is not perfectly round, and has deviations from its average radius with rms value $\delta R_{\text {aperture }}$, then the rms deviation from round at the detector will be $(M+1) \delta R_{\text {aperture }}$; this will cause a broadening of the system $p r f$ by $[(M+1) / M] \delta R_{\text {aperture. }} \approx \delta R_{\text {aperture. }}$. If the deviation from roundness is due to ellipticity, either from bad fabrication or from the aperture not being exactly perpendicular to the capsule-detector direction, this effect is identified in the data processing and corrected. Other types of deviation from roundness can sometimes be identified in the data, but in the worst case, uncorrected deviations should have $\delta R_{\text {aperture }}<3 \mu \mathrm{m}$ as discussed in Sec. II.

Another source of error would come from an inaccurate value of the magnification $M$ (see Fig. 2 and its caption), which could be different from the expected value if $L_{1}$ or $L_{2}$ were not measured accurately. Since $M$ represents the ratio of structure size at the detector to structure size in the burn region, any error in $M$ would not cause a broadening of the prf but would cause a multiplicative error in the inferred burn region size. But since the radius of the aperture itself, $R_{\text {aperture, }}$ is measured in advance to better than $0.1 \%$, and since the radius of its projection on the detector, $(M+1) R_{\text {aperture, }}$ is determined as part of the data analysis, a self-consistent value of $M$ is determined from the data and is not subject to measurement error. 


\section{F. Net broadening}

The net result of these broadening effects can be estimated for the data analyzed in Fig. 6, where the measured $R_{\text {burn }}$ was shown to be $32 \mu \mathrm{m}$ with a statistical error of $\pm 1.6 \mu \mathrm{m}$. If we assume that the five sources of error discussed in Secs. A-E are independent, and that each generates a distortion in the system prf that is approximately Gaussian, then the net system prf would be a Gaussian whose radius $\mathrm{r}_{p r f}$ is the sum (in quadrature) of the individual radii. In this case, taking the upper limit for geometric error, $\mathrm{r}_{p r f} \approx \sqrt{3^{2}+1^{2}+0^{2}+3^{2}+3^{2}} \mu \mathrm{m} \approx 5 \mu \mathrm{m}$. The measured burn radius would then be the true burn radius added in quadrature with $\mathrm{r}_{\text {prf }}$. The error would be

$$
\delta R_{\text {burn }}=R_{\text {burn,measured }}-R_{\text {burn,true }} \approx 32 \mu \mathrm{m}-\sqrt{32^{2}-5^{2}} \mu \mathrm{m} \approx 0.4 \mu \mathrm{m},
$$

which is small compared to the statistical uncertainty of $1.6 \mu \mathrm{m}$.

\section{FIDELITY OF THE MEASURED BURN-REGION SIZES}

The following subsections describe experimental tests that demonstrate the repeatability and fidelity of the measured burn region sizes. In Sec. A, measurement consistency is experimentally demonstrated when six independent measurements of $R_{\text {burn }}$ made during the same symmetrically-driven implosion are compatible. Sec. B shows that the reconstructed burn profiles are independent of changes in the system magnification and the aperture size. Sec. C presents data suggesting that ambient fields do not affect the system response.

\section{A. Consistency of the measured burn profiles}

Figure 10a shows six separate measurements of $R_{\text {burn }}$ made during the same symmetrically-driven implosion (of a capsule with a $17-\mu \mathrm{m}$-thick plastic shell filled with a mixture of $6 \mathrm{~atm}$ of $\mathrm{D}_{2}$ and $12 \mathrm{~atm}$ of ${ }^{3} \mathrm{He}$ ). The implosion was imaged with three separate PCIS cameras fielded in TIMs 2, 3, and 4. (TIM3 is located $109^{\circ}$ from TIM2, and TIM4 is $101^{\circ}$ from TIM2 and $79^{\circ}$ from TIM3.) Each camera produced two measurements of $R_{\text {burn }}$ using the redundant-detector method described in Sec. II. The error bars in the figure represent statistical uncertainties, as discussed in Sec. III, and the set of six measurements is statistically consistent.

\section{B. Lack of dependence on imaging geometry}

A measurement of $R_{\text {burn }}$ should be independent of any changes in the geometric parameters of the camera, and this is demonstrated by data plotted in Fig. 10. Lack of dependence on aperture diameter is illustrated in Fig. 10b, which shows results from 5 successive implosions of capsules with $19-\mu$ m-thick plastic shells filled with 10.5 -atm $\mathrm{D}_{2}$ and $6-\mathrm{atm}{ }^{3} \mathrm{He}$. For each implosion, one TIM was equipped with a PCIS camera. The diameter of the aperture used for the first two and last two implosions was $2000 \mu \mathrm{m}$, while the diameter used for the third implosion was $600 \mu \mathrm{m}$. All results were statistically consistent with the mean value of $30 \mu \mathrm{m}$. Lack of dependence on the geometric magnification was demonstrated by simultaneous use of cameras with different $M$ on each of two implosions. In Fig. 10a data were taken with $M=12.5$ 
and $\mathrm{M}=20$, while in Fig. 10c $M=6$ and $M=9$ were used; in each case the two different values of $M$ resulted in the same measured $R_{\text {burn }}$.

\section{Lack of distortion by ambient fields}

Several plastic-shell implosions were used to look for possible systematic errors associated with stray fields. The two cameras used for shot 35004 were set up identically except that $L_{1}=5.4 \mathrm{~cm}$ for TIM3 and $L_{1}=3.7 \mathrm{~cm}$ for TIM4. If fields were significantly influencing protons in the region between the capsule and the aperture, we would expect to see a difference in the measured $R_{\text {burn }}$ from these two cameras. The results shown in Fig. 10c report essentially the same $R_{\text {burn }}$ for both cameras, suggesting that there were no serious field effects in the region before the aperture.

The three cameras used for shot 36730 were set up identically except that $L_{2}=36.6 \mathrm{~cm}$ for TIM2 and TIM3 while $L_{2}=58.6 \mathrm{~cm}$ for TIM4. If fields were affecting the protons between the aperture and the detector (a region surrounded by a conductor), we would expect to see differences in the measured values of $R_{\text {burn }}$. Figure 10a shows that all $R_{\text {burn }}$ results were consistent, suggesting that there were no serious field effects in the region behind the aperture.

Another recent experiment ${ }^{28}$ also provides evidence that fields do not dramatically alter the trajectory of $\mathrm{D}^{3} \mathrm{He}$ protons in the region past the aperture. In this test, a steel mesh $(50-\mu \mathrm{m}$ diameter wires, with 100 wires per inch) was placed directly in front of the imaging aperture. Examination of the proton-emission image revealed no distortions in the mesh-induced pattern, suggesting that fields are not significantly altering the trajectories of $14.7-\mathrm{MeV} \mathrm{D}^{3} \mathrm{He}$ protons.

\section{DISCUSSION}

Three proton core imaging cameras have been built and installed at OMEGA using the design discussed here, and they have now been used to study the spatial distributions of nuclear burn in a range of different implosion types using algorithms described elsewhere. ${ }^{11,12}$ An example of the analysis of the radial burn profile of a symmetric implosion was shown in Sec. III, with a $32-\mu \mathrm{m}$ burn radius. In another article, ${ }^{12}$ other types of implosions are studied and shown to have burn radii ranging from 20 to $80 \mu \mathrm{m}$; clear correlations are shown between the burn radius and implosion parameters (capsule shell material and thickness, fill gas pressure, and laser energy). In addition, 2-D reconstruction techniques have been used together with three nearly-orthogonal imaging cameras to study asymmetric burn regions and to show clear relationships between laser drive asymmetry, capsule shell asymmetry, and burn asymmetry. ${ }^{14}$ In Ref. 11 the issues of spatial resolution and statistical noise were studied theoretically. A range of calculations and measurements, described in Secs. IV and V, investigated other possible sources of error in measured burn region sizes. So far there is no evidence of any other errors that are as important as the statistical errors; more calculations and measurements, as well as comparisons with x-ray and neutron images, will be pursued in the future for further verification. In the meantime, proton emission imaging is providing interesting and useful data bearing on the understanding of capsule implosion dynamics and performance. A particularly nice feature of this imaging mode, which will be utilized in future studies, is the fact that the same reaction product studied here (the $\mathrm{D}^{3} \mathrm{He}$ proton) is also utilized by other diagnostics. Proton spectrometers ${ }^{16}$ measure the proton energy at different angles around a capsule, determining the areal density of the compressed shell from the energy loss, while the proton temporal 
diagnostic ${ }^{29,30}$ measures the time evolution of the burn. The spatial distribution of $\mathrm{D}^{3} \mathrm{He}$ burn can therefore be combined with the angular distribution of areal density in the colder shell material and the time evolution of burn, as well as other types of diagnostic measurements, for a more complete picture of implosion dynamics.

\section{ACKNOWLEDGEMENTS}

The authors express their gratitude to the OMEGA engineers and operations crew who supported these experiments. In addition, we would like to personally thank Candice Culligan, and Jocelyn Schaeffer for their continuous help. This work has been supported in part by LLE (Subcontract No. P0410025G) and LLNL (Subcontract No. B313975), and by the U.S. Department of Energy Office of Inertial Confinement Fusion (Grant No. DE-FG03-99DP00300) and under Cooperative Agreement No. DE-FC03-92SF19460, the University of Rochester, and New York State Energy Research and Development Authority. 


\section{REFERENCES}

1. J. Nuckolls, L. Wood, A. Theissen et al., Nature 239, 139 (1972).

2. S. E. Bodner, D. G. Colombant, J. H. Gardner et al., Phys. Plasmas 5, 1901 (1998).

3. K. A. Nugent and B. Luther-Davies, J. Appl. Phys. 58, 2508 (1985).

4. D. Ress, R. A. Lerche, R. J. Ellis et al., Rev. Sci. Instrum. 59, 1694 (1988).

5. R. A. Lerche, D. Ress, R. J. Ellis et al., Laser Part. Beams 9, 99 (1991).

6. C. R. Christensen, D. C. Wilson, C. W. Barnes et al., Phys. Plasmas 11, 2771 (2004).

7. L. Disdier, R. A. Lerche, J. L. Bourgade et al., Rev, Sci. Instrum. 75, 2134 (2004).

8. Y. -W. Chen, M. Yamanaka, N. Miyanaga et al., Opt. Commun. 73, 227 (1989).

9. M. Nakai, M. Yamanaka, H. Azechi et al., Rev. Sci. Instrum. 61, 3235 (1990).

10. A. P. Fews, P. A. Norreys, F. N. Beg et al., Phys. Rev. Lett. 73, 1801 (1994).

11. F. H. Séguin, J. L. DeCiantis, J. A. Frenje et al., Rev. Sci. Instrum. 75, 3520 (2004).

12. F. H. Séguin, J. L. DeCiantis, J. A. Frenje et al., "Measured dependence of nuclear burn region size on implosion parameters in inertial confinement fusion experiments", submitted to Phys. Plasmas.

13. J. L. DeCiantis, MS Thesis, Massachusetts Institute of Technology (2005).

14. F. H. Séguin et al., F. H. Séguin, J. L. DeCiantis et al., Bull. Am. Phys. Soc. 49, 63 (2004); Bull. Am. Phys. Soc. 50, 114 (2005); and "Measured Effects of drive asymmetry and shell asymmetry on nuclear burn region symmetry in direct-drive ICF implosions" (to be submitted to Phys. Plasmas).

15. T. R. Boehly, D. L. Brown, R. S. Craxton et al., Opt. Commun. 133, 495 (1997).

16. F. H. Séguin, J. A. Frenje, C. K. Li et al., Rev. Sci. Instrum. 74, 975 (2003).

17. A series of burn images have been obtained from thin glass implosions involving less than $23 \mathrm{~kJ}$ of laser energy, ${ }^{12}$ several thick plastic shell implosions involving $18 \mathrm{~kJ}$ and $30 \mathrm{~kJ}$ of laser energy, ${ }^{12,14}$ and a few implosions involving different shaped laser pulses. ${ }^{13}$

18. T. R. Boehly, V. A. Smalyuk, D. D. Meyerhofer et al., J. Appl. Phys. 85, 3444 (1999).

19. C. K. Li and R. D. Petrasso, Phys. Plasmas 2, 2460 (1995).

20. C. K. Li and R. D. Petrasso, Phys. Rev. Lett. 70, 3059 (1993).

21. C. K. Li, D. G. Hicks, F. H. Séguin et al., Phys. Plasmas 7, 2578 (2000).

22. F. H. Séguin, C. K. Li, J. A. Frenje et al., Phys. Plasmas 9, 3558 (2002).

23. SRIM, a code for calculations of the Stopping and Ranging of Ions in Matter, Jan. 1 2004, Version 2003.26 (J. F. Ziegler and J. P. Biersack).

24. D.E. Post, R.V. Jensen, G.B. Tarter et al., Atomic Data and Nuclear Data Tables 20, No. 5 (1977).

25. J. D. Jackson, Classical Electrodynamics (John Wiley \& Sons, Inc., New York, NY, 1999).

26. D.G. Hicks, C. K. Li, F. H. Séguin et al., Phys. Plasmas 8, 606 (2001).

27. D.G. Hicks, C. K. Li, F. H. Séguin et al., Phys. Plasmas 7, 5106 (2000).

28. C. K. Li et al., Bull. Am. Phys. Soc. 50, 266 (2005).

29. J. A. Frenje et al., Phys. Plasmas 11, 2798 (2004).

30. V. Yu Glebov et al., Rev. Sci. Instrum., accepted. 


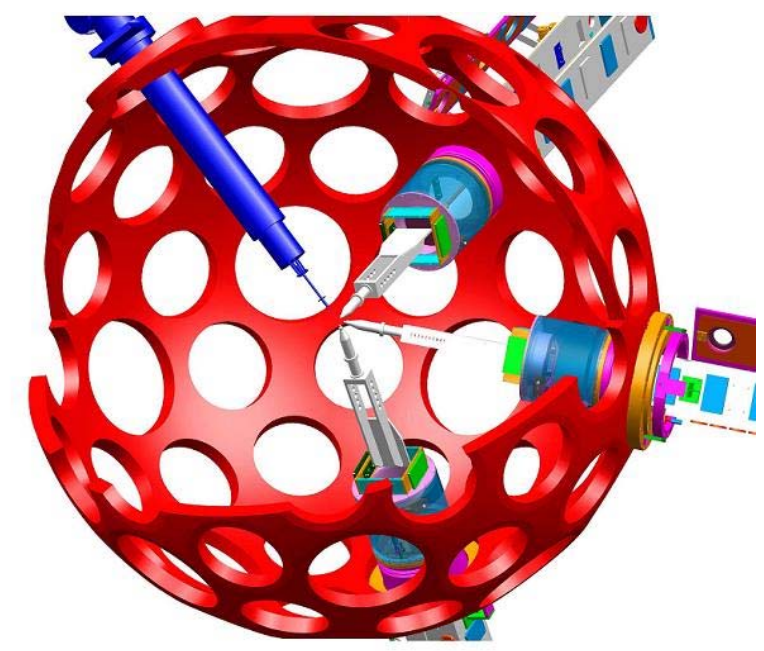

FIG. 1. (Color) Schematic of the OMEGA target chamber with three cameras for imaging a capsule implosion. The capsule is positioned at target-chamber center by the positioning system located at 11 o'clock. The three structures at 1,4 , and 5 o'clock are the PCIS cameras, located approximately orthogonal to each other. This orthoganality is especially important for studying asymmetric burn. 


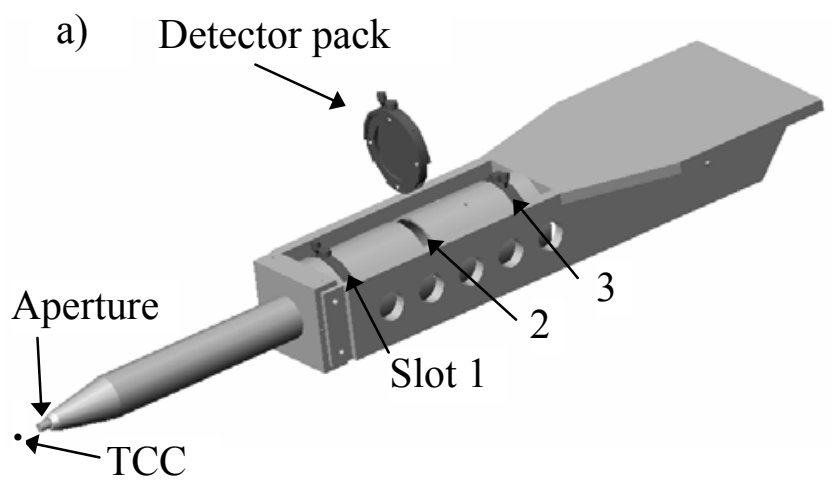

b) Nuclear burn region
(at TCC) Detector

FIG. 2. (a) Illustration of the proton-emission imaging hardware. The capsule implosion is located at target chamber center (TCC), which is about $3 \mathrm{~cm}$ from the imaging aperture. A detector pack may be positioned in one of three available slots $(34,47$, and $59 \mathrm{~cm}$ from the aperture), providing geometric magnification of capsule structure $\left(M \equiv L_{2} / L_{1}\right)$ of $12.5,16$, or 20. The aperture consists of a $0.2-\mathrm{cm}$ hole in a $500-\mu \mathrm{m}$ thick tantalum substrate. The total length of the instrument is $93 \mathrm{~cm}$. (b) A line diagram illustrates camera dimensions. 

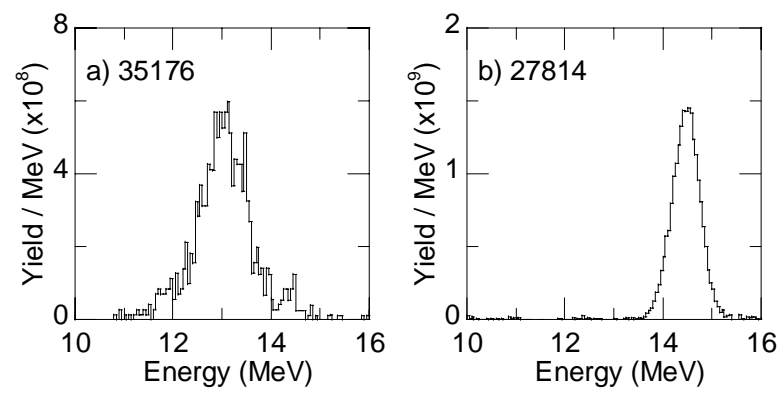

FIG. 3 For proton-emission imaging, filters are used to reduce the incident proton energies to the range $0.5-8 \mathrm{MeV}$ (where the CR-39 detectors are 100\% efficient). Different implosion types result in somewhat different proton energies, as shown here, and filters must be chosen accordingly. (a) A capsule with 20- $\mu$ m-thick $\mathrm{CH}$ shell (shot 35176). (b) A capsule with 2.3- $\mu \mathrm{m}-$ thick glass shell (shot 27814). These spectra were measured with "Wedge-Range-Filter" proton spectrometers. ${ }^{16}$

a)

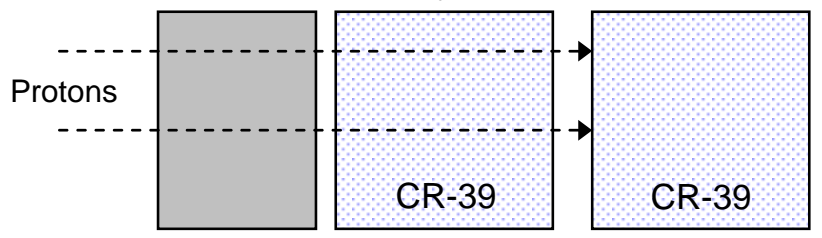

b)

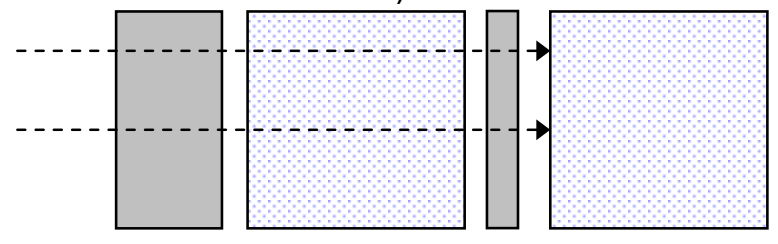

FIG. 4. Two examples of how CR-39 detectors and filters can be stacked for redundant "frontback" particle detection. (a) Protons lose enough energy while passing through the filter and the first piece of CR-39 so that their energies are in the range of 100\% detection efficiency and they leave tracks on both the back side of the first piece and the front side of the second piece. This provides protection against the possibility that either detector has a defect. (b) Protons lose enough energy while passing through the first filter and the first piece of CR-39 so that they leave tracks on the backside of the first piece. They lose slightly more energy while passing through another thin filter, leaving tracks on the front side of the second piece. This provides some protection against the possibility that the proton energy was too low at the second piece; the higher energy at the back of the first piece may then be optimal. 


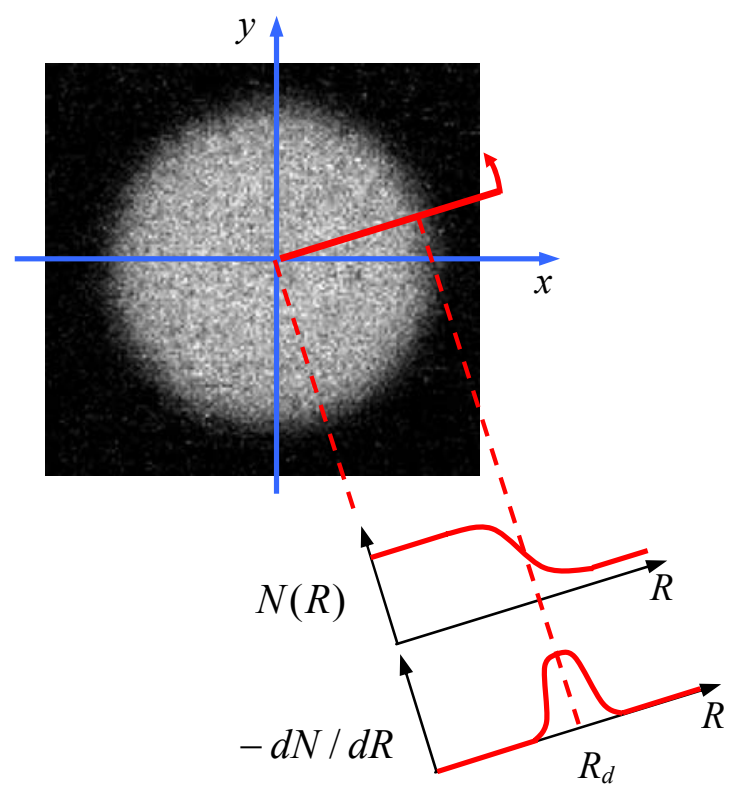

FIG. 5. (Color online) Illustration of the relationship between a raw penumbral image (brightness is proportional to the number of protons $N$ per unit area on the detector) and its radial derivative. In the method implemented here, only the azimuthal average of this derivative is used. In the more general case, where asymmetric burn regions are studied, the angular variation of the radial derivative is used. ${ }^{11}$ 

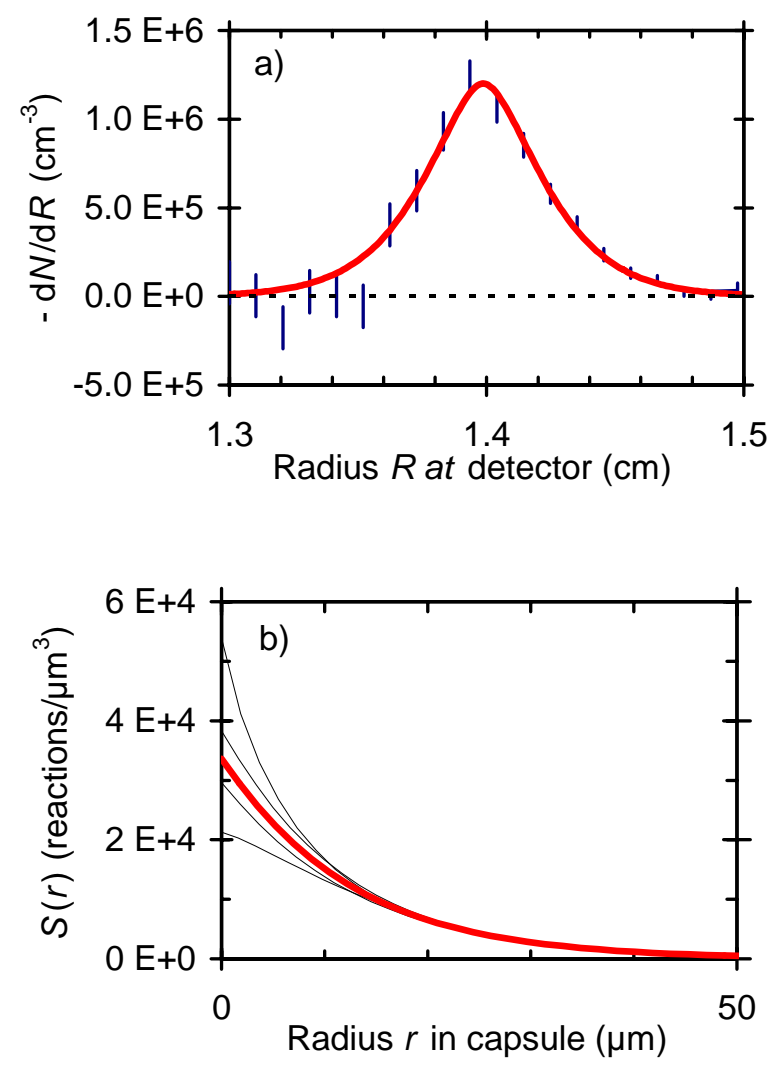

FIG. 6. (Color online) (a) The radial derivative of the azimuthally averaged detected proton density $(\mathrm{d} N / \mathrm{d} R)$ as a function of detector radius for OMEGA implosion 36730 . The data were obtained with one of three proton-emission imaging cameras, and the implosion involved a 17$\mu \mathrm{m}$ plastic shell and an 18 -atm $\mathrm{D}^{3} \mathrm{He}$-gas fill. The statistical error bars are larger (smaller) toward the left (right) of the peak where $\mathrm{d} N / \mathrm{d} R$ represents a small difference between two large (small) numbers. The solid line, representing the best fit to the data, corresponds to the radial burn profile ( ${ }^{3} \mathrm{He}$ reactions per unit volume) shown in (b) with $R_{\text {burn }}=32 \mu \mathrm{m}$. The error envelope shown by the fine lines in (b) was generated by letting the total $\chi^{2}$ of the fit increase by 1 ; although the uncertainty in the shape is significant, the statistical uncertainty in $R_{\text {burn }}$ is only $\pm 1.6 \mu \mathrm{m}$. The value of the shape parameter $p$ was $1.9 \pm 0.4$. 


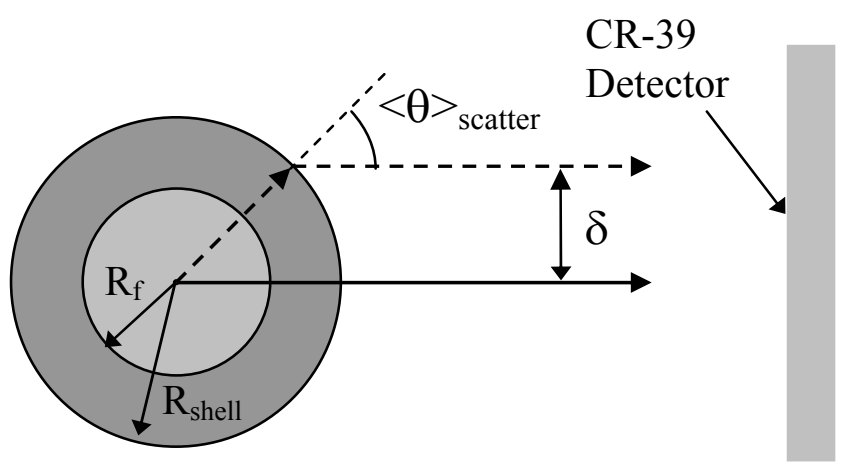

FIG. 7. Illustration of the assumed geometry for estimating the degree of proton scattering in shell material. The radius of the fuel region, $\mathrm{Rf}$, was taken to be $45 \mu \mathrm{m}$; the outer radius of the shell, Rshell, was $80 \mu \mathrm{m}$. Two proton paths originating from a point source are illustrated. The solid line represents the path taken by unscattered protons; the dotted line represents the worstcase path of scattered protons. The average scattering angle $\langle\theta\rangle$ scatter was estimated both for cold $\mathrm{CH}$ and for a fully-ionized $\mathrm{CH}$ plasma shell. The results were $\sim 1^{\circ}$ and $2^{\circ}$, respectively, producing corresponding source broadening, $\delta$ of $\sim 1 \mu \mathrm{m}$ and $3 \mu \mathrm{m}$. Both are negligible compared to typical measured burn radii of $\sim 30 \mu \mathrm{m}$. 

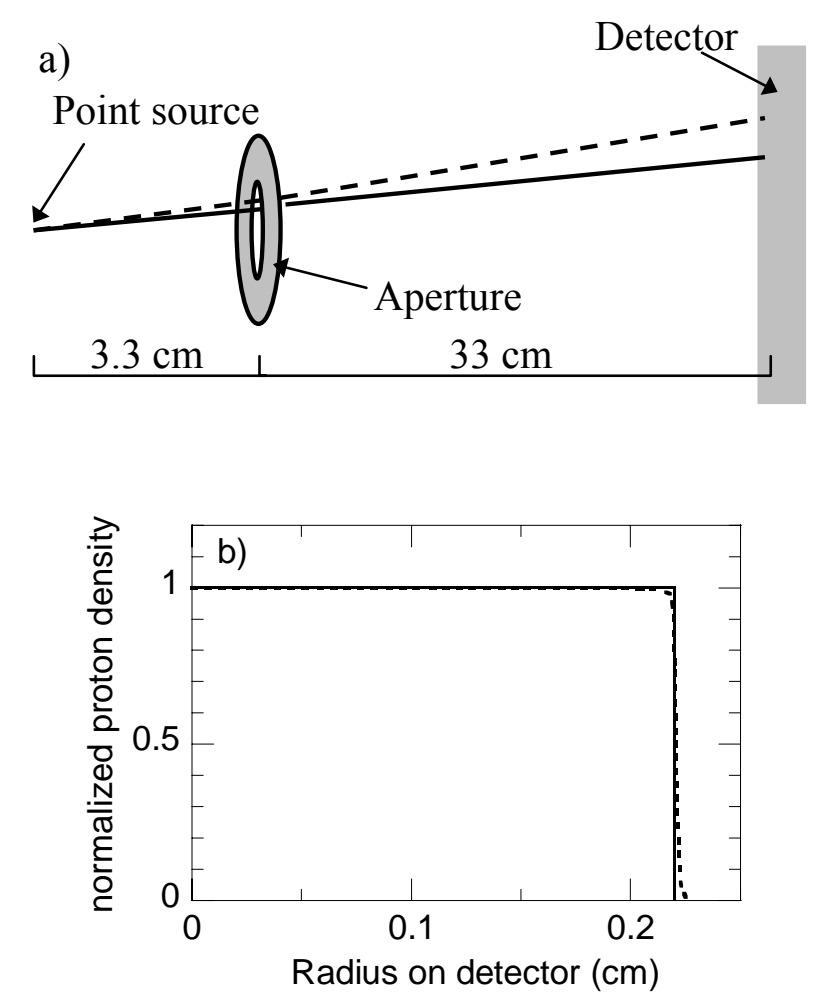

FIG. 8. (a) Illustration of the effect of electric fields on proton trajectories. The dashed line schematically represents the proton path when a $400-\mu \mathrm{m}$-diameter aperture carries a potential of $1 \mathrm{MV}$ relative to the source (an overestimate of realistic values), while the solid line represents the proton path when no potential is present. Calculations were performed to estimate the image broadening of a point source. (b) The simulated proton density striking the detector for the case of no potential (solid line) and a 1-MV potential (dashed line). The result was a slight outward shift of the image and a broadening of the point source by $\sim 1 \mu \mathrm{m}$ This broadening is small compared to a typical burn radius of $\sim 30 \mu \mathrm{m}$. 

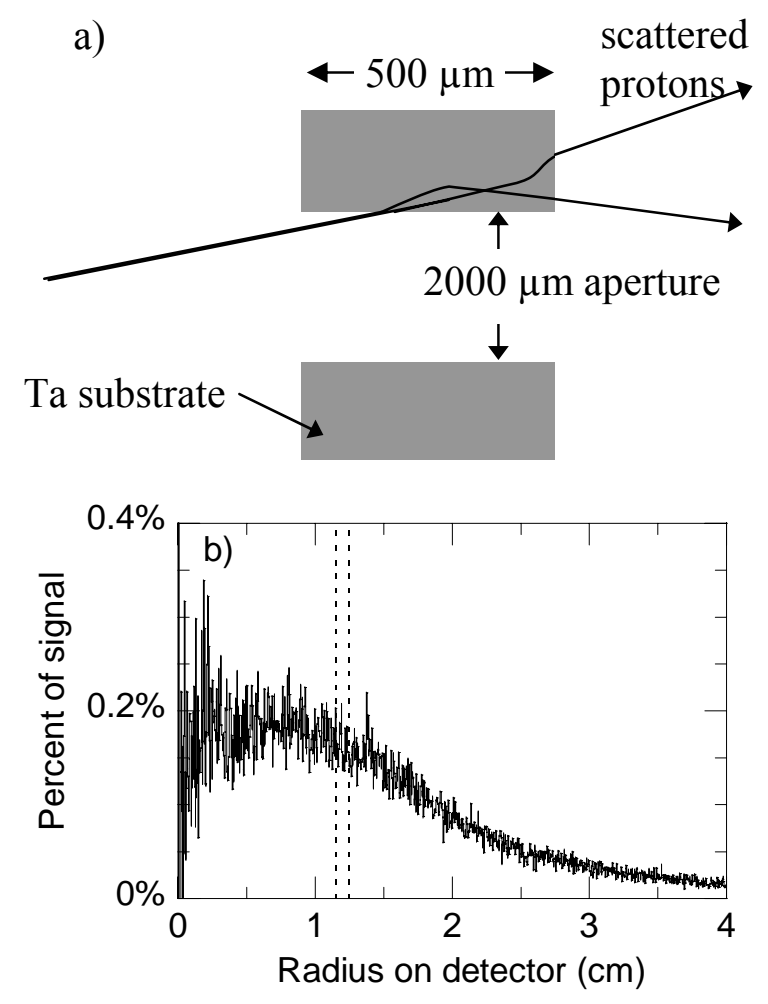

FIG. 9 (a) Cartoon of a cross sectional view of an aperture, defined as a 2000- $\mu$ m diameter hole in a 500- $\mu \mathrm{m}$ thick tantalum substrate. Not shown are the source and the detector, located $3 \mathrm{~cm}$ to the left and $33 \mathrm{~cm}$ to the right, respectively. As seen in the figure, the geometry dictates that protons may encounter less than $500 \mu \mathrm{m}$ of Ta. If the amount of Ta encountered is sufficiently small, the protons will exit the tantalum and may broaden the image. (b) The simulated ratio of scattered proton density to unscattered signal proton density on the detector. The dotted lines represent the approximate location of an image penumbra from a $\sim 30 \mu \mathrm{m}$ source. The calculated broadening of the point source was $<<1 \mu \mathrm{m}$. 

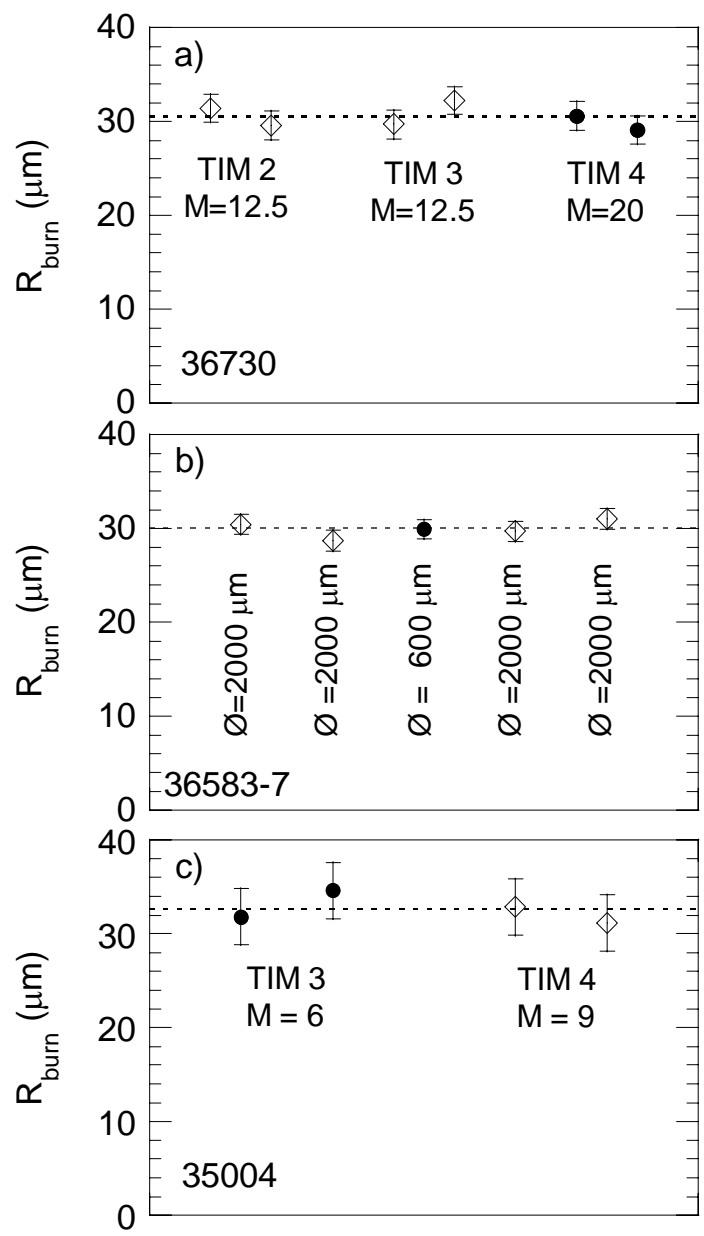

FIG. 10. Data illustrating that $R_{\text {burn }}$ measurements are repeatable and independent of imaging geometry. (a) An imploded capsule with a 17 - $\mu \mathrm{m}$-thick plastic shell and 18 -atm $\mathrm{D}^{3} \mathrm{He}$ gas-fill (shot 36730) was imaged by three nearly orthogonal imaging cameras with different aperture-todetector distances, located in TIMs 2, 3, and 4. The redundant-detector method was used to generate two data sets for each camera. The geometric magnification $(M)$ of each camera is listed. The data show that different cameras and different aperture-to-detector distances produce the same $R_{\text {burn }}$. Values were all statistically compatible with the mean (indicated by the dotted line). (b) A single camera imaged a series of $19-\mu \mathrm{m}$ thick plastic shells filled with $10.5 \mathrm{~atm} \mathrm{D}_{2}$ and $6 \mathrm{~atm}{ }^{3} \mathrm{He}$. The aperture diameter $(\varnothing)$ used for each camera is indicated; $R_{\text {burn }}$ was unaffected by the change in aperture size. (c) An implosion of a $20-\mu \mathrm{m}$-thick plastic shell with 18 -atm $\mathrm{D}^{3} \mathrm{He}$ gas fill was imaged by two cameras having different capsule-to-aperture distances, placed in TIMs 3 and 4 . The separately inferred values of $R_{\text {burn }}$ were statistically compatible. 